\title{
Wrangling Messy CSV Files by Detecting Row and Type Patterns
}

\author{
Gerrit J.J. van den Burg${ }^{1}$, Alfredo Nazábal ${ }^{1}$, and Charles Sutton ${ }^{1,2,3}$ \\ ${ }^{1}$ The Alan Turing Institute, London, UK \\ ${ }^{2}$ Google, Inc. Mountain View, CA, USA \\ ${ }^{3}$ School of Informatics, The University of Edinburgh, UK
}

November 29, 2018

\begin{abstract}
It is well known that data scientists spend the majority of their time on preparing data for analysis. One of the first steps in this preparation phase is to load the data from the raw storage format. Comma-separated value (CSV) files are a popular format for tabular data due to their simplicity and ostensible ease of use. However, formatting standards for CSV files are not followed consistently, so each file requires manual inspection and potentially repair before the data can be loaded, an enormous waste of human effort for a task that should be one of the simplest parts of data science. The first and most essential step in retrieving data from CSV files is deciding on the dialect of the file, such as the cell delimiter and quote character. Existing dialect detection approaches are few and non-robust. In this paper, we propose a dialect detection method based on a novel measure of data consistency of parsed data files. Our method achieves $97 \%$ overall accuracy on a large corpus of realworld CSV files and improves the accuracy on messy CSV files by almost $22 \%$ compared to existing approaches, including those in the Python standard library.
\end{abstract}

Keywords — Data Wrangling, Data Parsing, Comma Separated Values 
CSV is a textbook example of how not to design a textual file format.

— The Art of Unix Programming, Raymond (2003).

\section{Introduction}

The goal of data science is to extract valuable knowledge from data through the use of machine learning and statistical analysis. Increasingly however, it has become clear that in reality data scientists spent the majority of their time importing, organizing, cleaning, and wrangling their data in preparation for the analysis (Dasu and Johnson, 2003; Lohr, 2014; Kandel et al., 2011; Crowdflower, 2016; Kaggle, 2017). Collectively this represents an enormous amount of time, money, and talent. As the role of data science is expected to only increase in the future, it is important that the mundane tasks of data wrangling are automated as much as possible. It has been suggested that one reason that data scientists spent the majority of their time on data wrangling issues is due to what could be called the double Anna Karenina principle: "every messy dataset is messy in its own way, and every clean dataset is also clean in its own way" (Sutton et al., 2018). ${ }^{1}$ Because of the wide variety of data quality issues and data formats that exist ("messy in its own way"), it is difficult to re-use data wrangling scripts and tools, perhaps explaining the manual effort required in data wrangling.

This problem can be observed even in the earliest and what might be considered the simplest stages of the data wrangling process, that of loading and parsing the data in the first place. In this work, we focus as an example on comma-separated value (CSV) files, which despite their deceptively simple nature, pose a rich source of formatting variability that frustrates data parsing. CSV files are ubiquitous as a format for sharing tabular data on the web; based on our data collection, we conservatively estimate that GitHub.com alone contains over 19 million CSV files. Open government data repositories make increasingly more datasets available and often present their data in CSV format. ${ }^{2}$ Advantages of CSV files include their simplicity and portability, but despite some standardization effort (RFC 4180; Shafranovich, 2005), a wide variety of subtly incompatible variations of CSV files exist, including the 39 different dialects among the CSV files in our data. For example, we observe that values can be separated by commas, semicolons, spaces, tabs, or any other character, and can be surrounded by quotation marks or apostrophes to guard against delimiter collision or for no reason at all. Because so many variations of the CSV file format exist, manual inspection is often required before a file can be loaded into an analysis program.

Every messy CSV file is indeed messy in its own way, as we see from analysing a corpus of over 18,000 files in our study (Section 5). Figure 1 illustrates a few of the variations and problems that real-world CSV files exhibit by presenting simplifications of real-world files encountered during this study. Figure 1(a) illustrates a normal CSV file that uses comma as the delimiter and has both empty and quoted cells. Figure 1(b) shows a variation encountered in this study that uses the caret symbol as delimiter and the tilde character as quotation mark. Next, Figure 1(c) illustrates an ambiguous CSV file: the entire rows are surrounded with quotation marks, implying that the correct interpretation is a single column of strings. However, if the quotation marks are stripped a table appears where values are separated by the comma. Figure 1(d) illustrates a file with comment lines that have the hash symbol as a prefix. Figure 1(e) is adapted from Döhmen et al. (2017) and illustrates a file where multiple choices for the delimiter result in the same number of rows and columns (the semicolon, space, comma, and pound sign all yield three columns). Finally, Figure 1(f) illustrates a number of issues simultaneously: quote escaping both

\footnotetext{
${ }^{1}$ This problem is related to the principle of the fragility of good things (Arnold, 2003).

${ }^{2}$ Mitlöhner et al. (2016) survey 200,000 CSV files from open government data portals.
} 
In the formalism for CSV parsing we present in this paper, we distinguish two aspects of what makes the format of a file "messy". First, CSV files have a dialect that captures how tabular data has been converted to a text file. This dialect contains the delimiter, the quote character, and the escape character. Knowing this dialect is the first step to importing the data from the file as it provides all the information necessary to convert the raw text file into a matrix of strings. Second, CSV files may contain headers, have additional comment text (potentially indicated by a prefix character), contain multiple tables, encode numeric data as strings, or contain any of the myriad other issues that affect how the tabular data is represented. Thus while the dialect is sufficient to recover a matrix of strings from the file, the full CSV parsing problem contains additional steps to recover the original data.

The problem of dialect detection, which is our focus here, is a subproblem of CSV parsing in general. The broader CSV parsing problem includes file encoding detection, dialect detection, table detection, header detection, and cell type detection. We restrict ourselves to dialect detection for two reasons. First, while encoding detection for CSV files may be slightly different than the general encoding detection problem due to different character frequencies, we consider this problem sufficiently solved for our purposes and employ the method of Li and Momoi (2001). With this in mind, the next problem to solve is that of dialect detection. Second, the vast majority of CSV files have only a single table (Mitlöhner et al., 2016) and thus solving dialect detection is a more pressing issue than table detection.

A CSV file is created by some CSV "formatter" (either a program or a human) using a particular dialect. To load the file and decide on the dialect a human analyst would use their understanding of the CSV format and its many variations, an understanding of what the data embedded in the file represents, and potentially years of experience of dealing with messy CSV files. Automating this process is non-trivial: we receive a text file from an unknown source, created with an unknown formatter using an unknown dialect, that contains unknown data, and are asked to choose the parameters that allow a faithful reconstruction of the original data. Considering the large number of unknowns in this process, it may not be surprising that many programming languages do not attempt to automate this process at all and instead leave the user to deal with the issue. It may also explain why only very little attention has been paid to this problem in the literature.

This paper is structured as follows. In Section 2 we present an overview of related work on both table detection and CSV parsing. Next, Section 3 gives a formal mathematical description of CSV parsing that presents dialect detection as an inverse problem. Our proposed data consistency measure for dialect detection is presented in Section 4. Results of a thorough comparison of our method with the few existing alternatives are presented in Section 5. Section 6 concludes the paper.

\section{Related Work}

Only very few publications have paid any attention to the problem of CSV parsing. Mitlöhner et al. (2016) explore a large collection (200K) of CSV files extracted from open data platforms of various governments. The authors use a number of heuristics to explore and parse the files. Dialect detection is done with the Python built-in CSV sniffer mentioned above, but the accuracy of this detection is not evaluated. Despite the extensive analysis of this large corpus of CSV files, the authors do not present a novel CSV parser or dialect detection method based on their heuristics.

A recent paper that does present a novel CSV parser and specifically addresses the problem of messy CSV files is that of Döhmen et al. (2017). They present HypoParsr, a so-called "multi- 
hypothesis" CSV parser that builds a tree of all possible parser configurations and subsequently scores each configuration with a heuristic method. The authors evaluate their parser on 64 files with known ground truth and a corpus of 15,000 files from the UK open government portal without ground truth. While they achieve a high parsing "accuracy" on the latter corpus of CSV files, this does not necessarily reflect a correct parsing of the CSV files due to the absence of ground truth. In our preliminary analysis of this parser we observed that its implementation is rather fragile and does not return a parsing result for many messy CSV files. Moreover, on several files from the UK open government portal we observed that while the parser returned without error, it did in fact quietly drop several rows from the result. This illustrates the difficulty of the CSV parsing problem: without a large enough set of real-world messy CSV files it is hard to create a parser that is robust against all the problems and variations that can occur.

A closely related subject to CSV parsing and dialect detection is that of detecting tables in free text. Early work on this topic includes the work of $\mathrm{Ng}$ et al. (1999) who address the problem of identifying the location of a table in free text. They use a decision tree classifier based on features extracted from the content of the text, such as whether or not a character is next to whitespace, or a character is a symbol, etc. Later work by Pinto et al. (2003) applies a similar strategy for constructing features but instead uses conditional random fields and expands the problem by also identifying the semantic role of each row in the identified table (i.e. header, data row, etc.). However, CSV parsing differs from identifying tables in free text because CSV files have more structure and flexibility than free text tables. CSV files use a specific character to delimit cells and can employ the quoting mechanism to let cells span multiple lines and guard against delimiter collision. This explains why the methods mentioned above for detecting tables in free text cannot be readily applied to CSV parsing.

Other work related to CSV parsing is the work on DeExcelerator by Eberius et al. (2013). This work focuses mainly on spreadsheets in Excel format, but can also handle CSV files when the correct dialect is provided by the user. The DeExcelerator program then extracts tables from the spreadsheet and in the process performs header recognition, data type recognition, and value extrapolation, and other operations based on heuristic rules. In follow-up work, Koci et al. (2016) present a method for classifying the role of each cell in the spreadsheet (i.e. attribute, data, header, etc.) using surface-level features derived from the formatting of the text and classification methods such as decision trees and support vector machines. While the DeExcelerator package offers no method for detecting the dialect of CSV files, the methods for table and header detection are suitable to the general CSV parsing problem outlined above.

More broadly, there is some work on data wrangling in particular that can be considered here. Fisher et al. (2008) present the PADS system for retrieving structured data from ad-hoc data sources such as log files of various systems. However, the authors explicitly mention that CSV files are not a source of ad-hoc data. Well-known work on the more general data wrangling problem is that of Kandel et al. (2011) on the Wrangler system. Here the human-machine interaction of data wrangling is considered and a system is proposed to assist the human analyst. In follow-up work, Guo et al. (2011) provide a method for automatically suggesting data transformations to the analyst and additionally provide a "table suitability metric" that quantifies how well a table corresponds to a relational format. The relational format is also known as attribute-value format or "tidy data" (Wickham, 2014) and it is often the goal of the data wrangling process. In our experiments we compare our method to the table suitability metric of Guo et al. (2011).

Finally, it is worth mentioning the few efforts that have aimed to solve the problem of CSV parsing at the source by proposing extensions or variations on the format that address some of the known issues with CSV files. A study on the use and future of CSV files on the web was performed by a working group of the World Wide Web Consortium (Tennison, 2016). One of the results of this working group is a proposal to provide metadata about a CSV file through an 
accompanying JSON (Crockford, 2006) description file (Tennison and Kellogg, 2015). A similar proposal is the CSV Dialect specification by Frictionless Data (2017) that recommends storing the dialect of the CSV file in a separate JSON object. While these recommendations could certainly address some of the issues of CSV parsing, it requires users to specify a second file and maintaining it next to the CSV file itself. Moreover, it does not address the issues of the existing messy CSV files. Alternatives such as the CSVY format (Rovegno and Fenner, 2015) propose to add a YAML (Evans, 2001) header with metadata. While this does combine the metadata and tabular data in a single file, it requires special import and export tools that may limit the adoption of these formats.

\section{Problem Statement}

We present a formal mathematical definition of CSV file parsing based on a generative process for how the file was constructed from the underlying data. This formal framework will define the language we subsequently use to present our methodology and will clarify the problem of CSV parsing in general. Within this framework dialect detection is presented as an inverse problem. This is a natural framing of the problem since we are interested in identifying the unknown dialect that was used to produce an observed outcome.

Let $\Sigma$ denote a finite alphabet of characters in some encoding $E$ and let $\Sigma^{*}$ be all the possible strings in this alphabet. ${ }^{3}$ Then a CSV file is an element $\mathbf{x} \in \Sigma^{*}$. A CSV file contains $N$ tuples $\mathbf{t}_{i}$ that represent elements of a product set, thus

$$
\mathbf{t}_{i} \in \mathcal{V}_{1} \times \mathcal{V}_{2} \times \cdots \times \mathcal{V}_{L_{i}}
$$

where the sets $\mathcal{V}_{j}$ are the domains of values in the tuples (Codd, 1970). These domains represent the sets that the values belong to, i.e. that floating point numbers have the domain $\mathcal{V}=\mathbb{R}$, for instance. Note that the length of a tuple is given by $L_{i}$. Since CSV files can contain comments or multiple tables we cannot assume that the length of tuples is constant throughout the file. The collection of all tuples in the file is given by the array $\mathbf{T}=\left[\mathbf{t}_{1}, \ldots, \mathbf{t}_{N}\right]$.

Next we define the dialect of CSV files. Conceptually, we can think of a CSV file as being generated by a formatter that takes the original data and converts it to its CSV file representation. The formatter has knowledge of the dialect $\boldsymbol{\theta}$ that has been used to create the CSV file, and it consists of two stages. In Stage 1 the formatter converts the data $\mathbf{T}$ to a string-only representation $\mathbf{C}$ where the domains in the tuples are the string domain (i.e. $\Sigma^{*}$ ). In Stage 2 this string-only representation is converted to a CSV file $\mathbf{x}$. When we wish to retrieve the data from the CSV file the information about both the formatter and the dialect has been lost. This leads us to the following definition.

Definition 3.1 (Dialect). Given a CSV file $\mathbf{x} \in \Sigma^{*}$, the dialect $\boldsymbol{\theta}$ represents all parameters needed for a one-to-one mapping from an array of tuples of strings $\mathbf{C}=\left[\mathbf{c}_{1}, \ldots, \mathbf{c}_{N}\right]$ to the file $\mathbf{x}$, thus

$$
f_{\boldsymbol{\theta}}(\mathbf{C})=\mathbf{x}
$$

Here $f_{\boldsymbol{\theta}}$ represents Stage 2 of the formatter. The inverse operation $f_{\boldsymbol{\theta}}^{-1}(\mathbf{x})=\mathbf{C}$ corresponds to converting the file $\mathbf{x}$ to an array of tuples of strings.

We may further define a function $g$ that is used in Stage 1 of the formatter to convert the data to the string representation,

$$
g(\mathbf{T})=\mathbf{C} .
$$

\footnotetext{
${ }^{3}$ The set $\Sigma^{*}$ is known as the Kleene closure (Kleene, 1956).
} 
The inverse of this function, $g^{-1}(\mathbf{C})$, returns the elements in the tuples to their original representation and is in practice often achieved through type casting. In practice one may additionally wish to specify an inverse operation $h^{-1}(\mathbf{T})$ that extracts from $\mathbf{T}$ the actual tabular data by identifying the location of rectangular tables in $\mathbf{T}$, detecting the presence of column headers, removing unnecessary comments, etc. This leads us to the following general definition.

Definition 3.2 (CSV Parsing). Let $\mathbf{x} \in \Sigma^{*}$ be a CSV file. The problem of CSV parsing then reduces to evaluating

$$
h^{-1}\left(g^{-1}\left(f_{\boldsymbol{\theta}}^{-1}(\mathbf{x})\right)\right) \text {. }
$$

Thus we see that the first step in parsing CSV files is indeed the detection of the correct dialect $\boldsymbol{\theta}$. In the remainder of this paper we will therefore focus our attention on this step. In the process we will often use the parsing function $f_{\boldsymbol{\theta}}^{-1}$ to parse the file for a given dialect. Although the exact definition of this function is not the main focus of the paper, it is worth mentioning that we base our implementation on the CSV parser in the Python standard library. Minor modifications of this code were made to simplify the dialect and handle certain edge cases differently, see Appendix B.5 for more details.

In this work we consider a dialect of three components: the delimiter $\left(\theta_{d}\right)$, the quote character $\left(\theta_{q}\right)$, and the escape character $\left(\theta_{e}\right)$. The delimiter is used to separate cells (i.e. values in the tuple), the quote character is used to enclose cells that may contain the delimiter, a newline character, or neither, and the escape character can be used to achieve nested quotation marks. Each of these parameters can be absent in a CSV file, thus $\theta_{d}, \theta_{q}, \theta_{e} \in \Sigma \cup\{\varepsilon\}$ with $\varepsilon$ the empty string (note that $\theta_{d}=\varepsilon$ for a file with a single column of data). ${ }^{4}$ While some existing parsers include other components of the dialect, such as whether or not nested quotes are escaped with double quotes, we found that it was possible to formulate $f_{\boldsymbol{\theta}}^{-1}$ with only the three components given above. Moreover, some of these other components are more accurately described as parameters to the functions $g^{-1}$ or $h^{-1}$.

\section{A Consistency Measure for Dialect Detection}

Having illustrated above the difficulty of dialect detection in general, we present here our solution based on two components: row length patterns and data type inference. The main idea that if a CSV file is parsed with the correct dialect rather than an incorrect one, the resulting tuples in the parsed data will appear more consistent. By consistency here we consider primarily two aspects: (a) the parsed rows should have similar length, and (b) cells within the tuples should have the same data type, such as integers or strings. To that end, we propose a measure of consistency over parsed data, which has two components, one that measures each of these two kinds of consistency. Then we search the space of possible dialects for the dialect in which the parsed file is most consistent. This aims to capture both aspects of how a human analyst would identify the dialect: searching for a character that results in regular row patterns and using knowledge of what real data "looks like".

More formally, we associate with each dialect $\boldsymbol{\theta}$ a consistency measure $Q(\mathbf{x}, \boldsymbol{\theta})=P(\mathbf{x}, \boldsymbol{\theta})$. $T(\mathbf{x}, \boldsymbol{\theta})$, where $P$ is a pattern score and $T$ is a type score. The estimate of the correct dialect is then obtained as

$$
\hat{\boldsymbol{\theta}}=\underset{\boldsymbol{\theta} \in \Theta_{\mathbf{x}}}{\arg \max } Q(\mathbf{x}, \boldsymbol{\theta}) .
$$

Algorithm 1 shows pseudocode for our search algorithm. The algorithm uses the fact that the type score $T(\mathbf{x}, \boldsymbol{\theta})$ is between 0 and 1 to speed up the search. In specific instances multiple

\footnotetext{
${ }^{4}$ Thus we restrict ourselves to CSV files where these parameters are all a single character. CSV files that use
} 


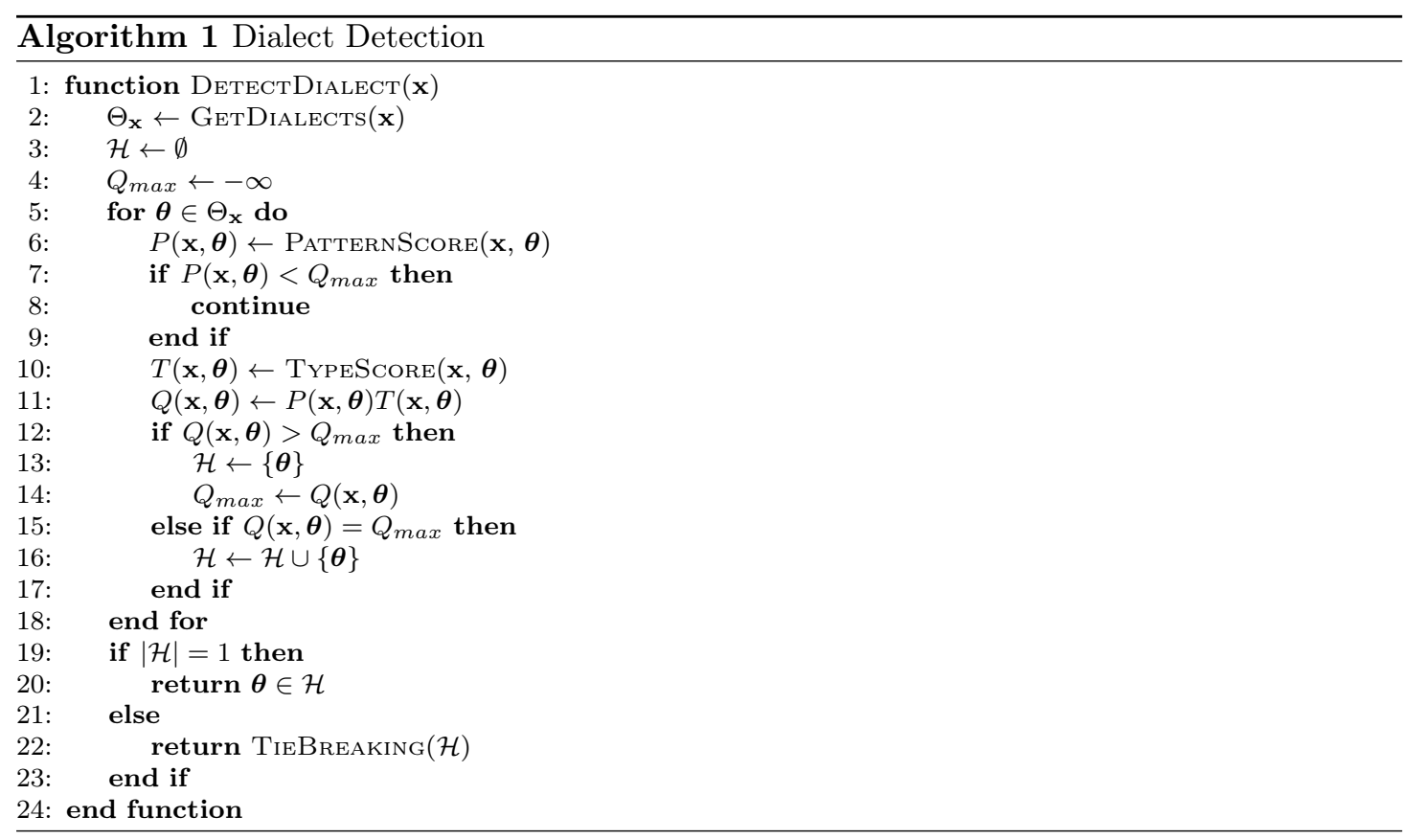

dialects can receive the same value for $Q(\mathbf{x}, \boldsymbol{\theta})$ due to limitations in the type score. However, some of these ties can be broken reliably and we will expand on this below as well.

\subsection{Pattern Score}

The pattern score is the main driver of the data consistency measure. It is based on the observation that since CSV files generally contain tables, we expect to find rows with the same number of cells when we select the correct dialect. For a given dialect we therefore parse the file and determine the number of cells in each row. This parsing step takes nested quotes and escape characters into account, and returns for each row a so-called row pattern. See Figure 2 for an illustration. The row patterns capture the repeated pattern of cells and delimiters and interpret quotes and escape characters where needed while abstracting away the content of the cells. $^{5}$ Notice how in Figure 2 the parsing result for $\boldsymbol{\theta}_{1}$ gives different row patterns than for $\boldsymbol{\theta}_{2}$ and $\boldsymbol{\theta}_{3}$. Each distinct row pattern $k$ has a length $L_{k}$, that is exactly one higher than the number of delimiters in the pattern. The number of times row pattern $k$ occurs in the file is called $N_{k}$ and the total number of distinct row patterns is denoted by $K$.

These properties are used to define the pattern score, as follows

$$
P(\mathbf{x}, \boldsymbol{\theta})=\frac{1}{K} \sum_{k=1}^{K} N_{k} \frac{L_{k}-1}{L_{k}} .
$$

The pattern score is designed to favour row patterns that occur often and those that are long, and also to favour fewer row patterns. The reasoning behind this is that when the correct row

\footnotetext{
multi-character delimiters do exist, but are extremely rare.

${ }^{5}$ This process interprets the quotes as defined by the dialect, but it can occur that a spurious quote character remains in the row pattern when an incorrect dialect is chosen. In this case, we consider patterns such as CDCQCDC - where $Q$ denotes the quote character, D the delimiter, and C any other character - to be distinct from CDCDC.
} 


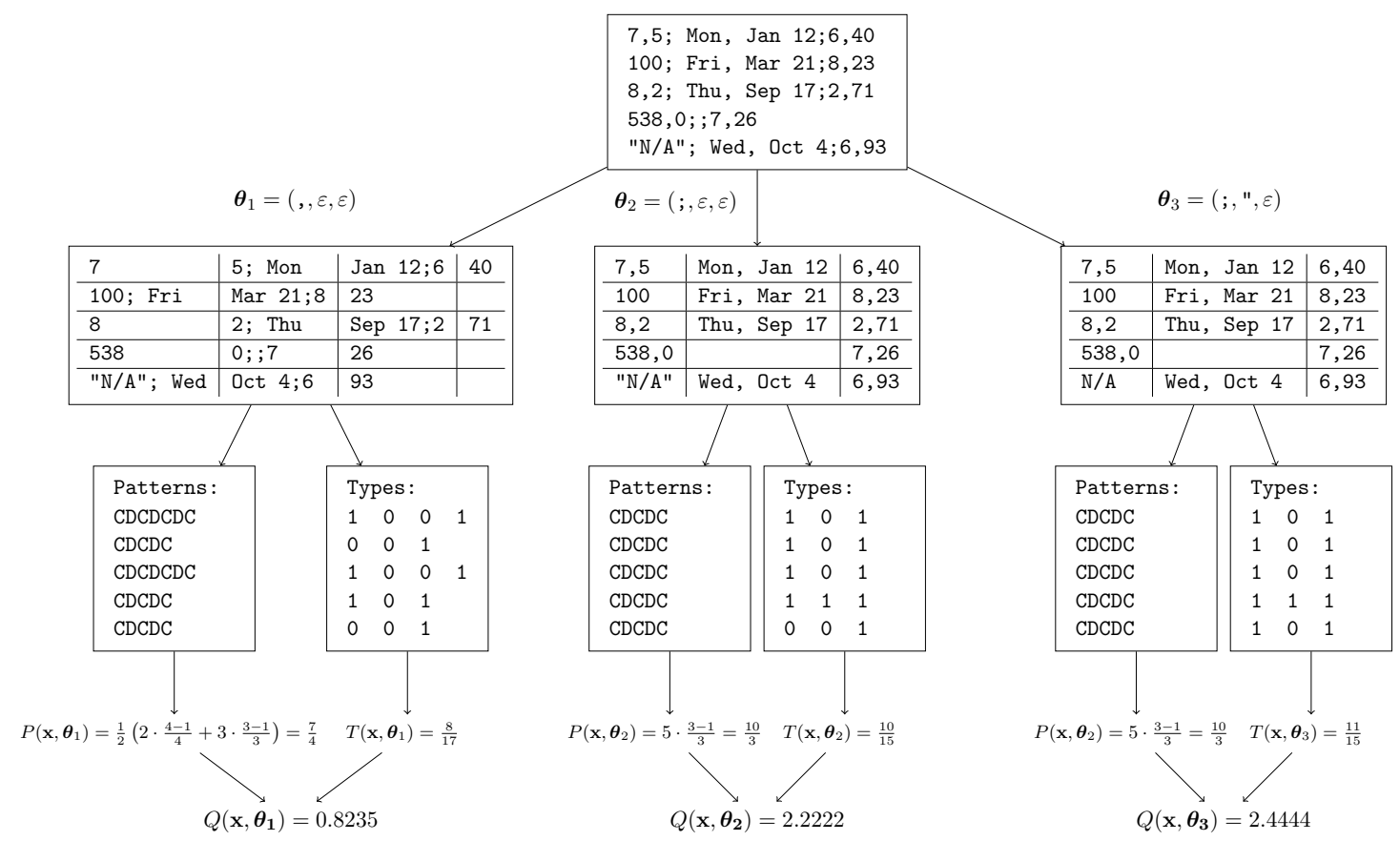

Figure 2. Illustration of the data consistency measure for different dialect on a constructed example. The figure shows how different dialects can result in different row patterns and pattern scores, as well as different type scores. The difference between $\boldsymbol{\theta}_{2}$ and $\boldsymbol{\theta}_{3}$ is due to the fact that the string $\mathrm{N} / \mathrm{A}$ belongs to a known type, but the string "N/A" does not.

pattern is chosen, we expect to observe few distinct row patterns, and that the ones that we do observe occur often (i.e. in tables). Figure 2 illustrates this aspect, as the correct delimiter yields a single row pattern that occurs throughout the entire file. By including the row length ratio we favour longer row patterns over shorter ones. This is included because a long pattern indicates a regular pattern of delimiters and cells, whereas a short pattern might indicate an incorrectly chosen delimiter.

While this function works well for CSV files that contain tables, it gives a value of 0 when the entire file is a single column of data. To handle these files in practice, we replace the numerator by $\max \left\{\alpha, L_{k}-1\right\}$ with $\alpha$ a small constant. The value of $\alpha$ must be chosen such that singlecolumn CSV files are detected correctly, while avoiding false positive results that assume regular CSV files are a single column of messy data. It was found empirically that $\alpha=10^{-3}$ achieves this goal well.

\subsection{Type Score}

While the pattern score is the main component of the data consistency measure, Figure 2 shows that the type score is essential to obtaining state-of-the-art results. The goal of the type score is to act as a proxy for understanding what the cells of the file represent, capturing whether a dialect yields cells that "look like real data". To do this, the type score measures the proportion of cells in the parsed file that can be matched to a data type from a set $\mathcal{T}$.

In our implementation, $\mathcal{T}$ includes empty cells, numbers in various formats, URLs, email addresses, percentages, currency values, times, dates, and alphanumeric strings (see Appendix A). 
We use regular expressions to detect these types, and denote the mapping from a string $\mathbf{z}$ to a type by $\mu(\mathbf{z})$. Then the type score is defined as

$$
T(\mathbf{x}, \boldsymbol{\theta})=\frac{1}{M} \sum_{i=1}^{N} \sum_{j=1}^{L_{i}} I\left[\mu\left(c_{i, j}\right) \in \mathcal{T}\right]
$$

where $M$ is the total number of cells in the parsing result $\mathbf{C}, c_{i, j}$ is the $j$-th value of tuple $\mathbf{c}_{i}$, and $I[\cdot]$ denotes the indicator function that returns 1 if its argument is true and 0 otherwise. While the type detection algorithm can identify many data types, it is infeasible to encapsulate all possible data types. Thus, the situation may arise where no type can be detected for any cell in the parsing result. This would result in a consistency measure $Q(\mathbf{x}, \boldsymbol{\theta})=0$, even though the pattern score may give high confidence for a dialect. To avoid this problem in practice, we replace $T(\mathbf{x}, \boldsymbol{\theta})$ by $\tilde{T}(\mathbf{x}, \boldsymbol{\theta})=\max \{\beta, T(\mathbf{x}, \boldsymbol{\theta})\}$, with $\beta=10^{-10}$.

\subsection{Tie breaking}

Unfortunately the value of $Q(\mathbf{x}, \boldsymbol{\theta})$ can be the same for different dialects even in the presence of the type score, due to the fact that the latter is necessarily incomplete. However in some cases these ties can be broken reliably. For example, if the same score is returned for two dialects that only differ in the quote character, then we can check whether or not the quote character has any effect on the parsing result. If it doesn't, then the quote character only occurs inside cells and does not affect the parsing outcome $\mathbf{C}=f_{\boldsymbol{\theta}}^{-1}(\mathbf{x})$. The correct solution is then to ignore the quote character. Similar tie breaking rules can be constructed for ties in the delimiter or the escape character.

\subsection{Potential Dialects}

In (5) we select the best dialect from a set of dialects $\Theta_{\mathbf{x}}$. It's worthwhile to expand briefly on how $\Theta_{\mathbf{x}}$ is constructed. While in general it is the product set of all potential delimiters, quote characters, and escape characters in the file, there are small optimizations that can be done to shrink this parameter space. Doing this speeds up dialect detection and reduces erroneous results.

Algorithm 2 presents pseudocode for the construction of $\Theta_{\mathbf{x}}$. The functions used in the algorithm are described in more detail in Appendix B. The algorithm proceeds as follows. As a preprocessing step, URLs are filtered from the file to remove potential delimiters that only occur in URLs. Next, potential delimiters are found with GetDelimiters by comparing the Unicode category of each character with a set of allowed categories and filtering out characters that are explicitly blocked from being delimiters. Next, potential quote characters are selected by matching with a set of allowed quote characters. Subsequently, the escape characters are those that occur at least once before a delimiter or quote character and that belong to the Unicode "Punctuation, other" category (The Unicode Consortium, 2018). Finally, $\Theta_{\mathbf{x}}$ is the product set of the potential delimiters, quote characters, and escape characters, with the exception that dialects with delimiters that never occur outside the quote characters in the dialect are dropped. This latter step removes more false positives from the set of potential dialects.

\section{Experiments}

In this section we present the results of an extensive comparison study performed to evaluate our proposed method and existing alternatives. Since variability in CSV files is quite high and the 


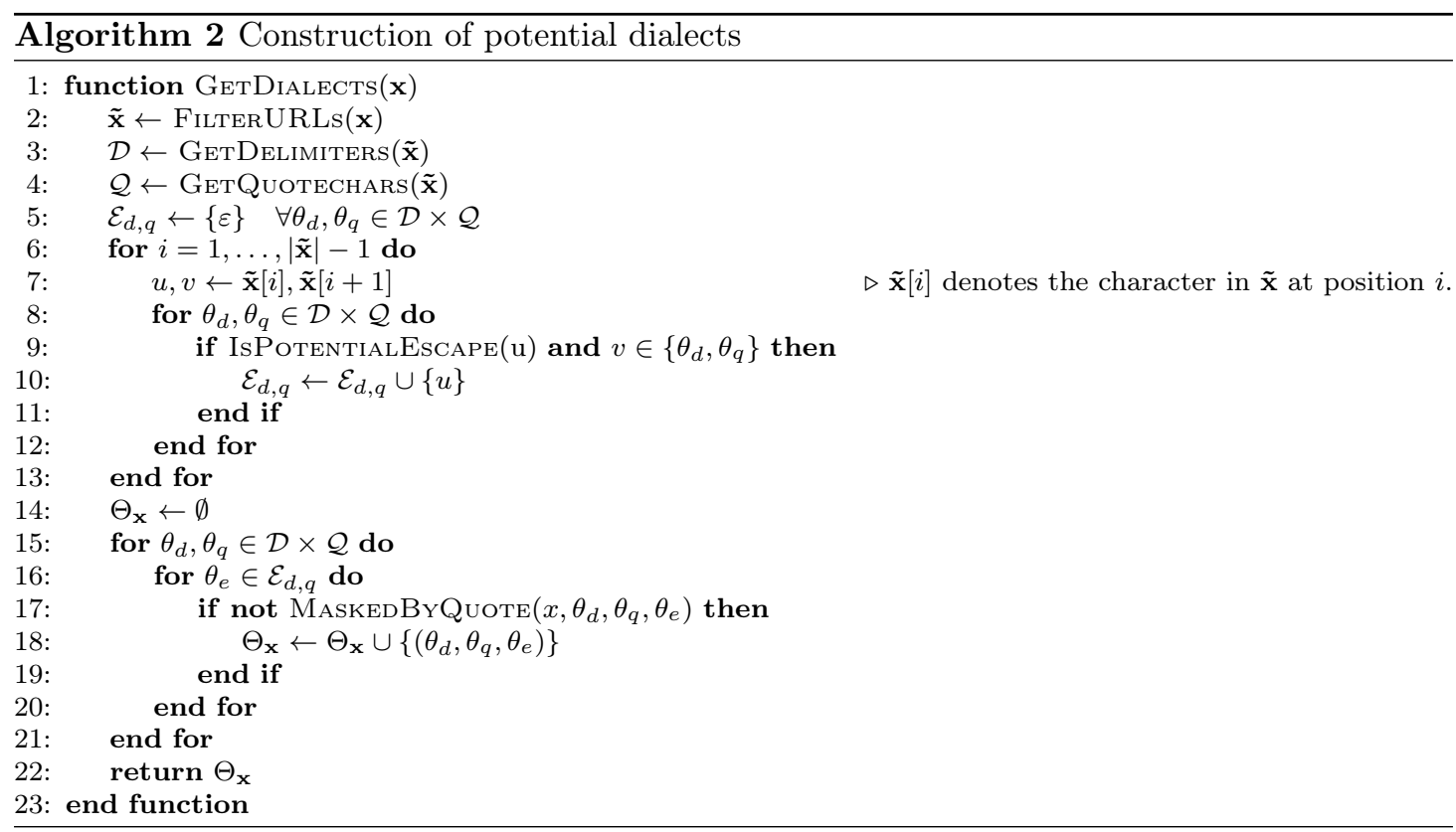

number of potential CSV issues is large, an extensive study is necessary to thoroughly evaluate the robustness of each method. Moreover, since different groups of users apply different formats, it is important to consider more than one source of CSV files.

Our method presented above was created using a development set of CSV files from two different corpora. The experimental section presents results from a comparison on an independent test set that was unknown to the authors during the development of the method. This split aims to avoid overfitting of our method and report the accuracy of our method accurately.

In an effort to make our work transparent and reproducible, we release the full code to replicate the experiments through an online repository. ${ }^{6}$ This will also enable other researchers to easily build on our work and allow them to use our implementations of alternative detection methods.

\subsection{Data}

Data was collected from two sources: the UK government open data portal (data.gov.uk) and GitHub (github.com). These represent different groups of users (government employees vs. programmers) and it is expected that we find differences in both the format and the type of content of the CSV files. Data was collected by web scraping in the period of May/June 2018, yielding tens of thousands of CSV files. From these corpora of CSV files we randomly sampled a development set (3776 files from UKdata and 4536 files from GitHub). These were used to develop and fine-tune the consistency measure presented above, and in particular were used to fine-tune the type detection engine.

When development of the method was completed, an independent test set was sampled from the two sources (5000 files from each corpus). This test set is similar to the development data, with one exception. During development we noticed that the GitHub corpus often contained multiple files from the same code repository. These files usually have the same structure and

\footnotetext{
${ }^{6}$ See https://github.com/alan-turing-institute/CSV_Wrangling.
} 
dialect, thus representing essentially a single example. Therefore, during construction of the test set a limit of one CSV file per GitHub repository was put in place. Thus we expect that the test set has greater variability and difficulty than the development set. It is worth emphasizing that the test set was not used in any way during the development of the method.

\subsection{Ground Truth}

To evaluate the detection method, we needed to obtain ground truth for the dialects of the CSV files. This is done through both automated and manual ways. The automated method is based on very strict functional tests that allow only simple CSV files with elementary cell contents. For instance, in one test we require that a file has a constant number of cells per row, no missing values, no nested quotes, etc. These automatic tests are sufficient to accurately determine the dialect of about a third of the CSV files. For the remainder of the files manual labelling was employed using a terminal-based annotation tool. Files that could not reasonably be considered CSV files were removed from the test set (i.e. HTML, XML, or JSON files, or simple text files without any tabular data). The same holds for files for which no objective ground truth could be established, such as files formatted similarly to the example in Figure 1(c). After filtering out these cases the test set contained 4873 files from GitHub.com and 4969 files from the UK government open data portal.

\subsection{Alternatives}

Since the dialect detection problem has not received much consideration in the literature, there are only a few alternative methods to compare to. We briefly present them here.

\subsubsection{Python Sniffer}

Python's built-in CSV module contains a so-called "Dialect Sniffer" that aims to automatically detect the dialect of the file ${ }^{7}$. This method detects the delimiter, the quote character, whether or not double quotes are used, and whether or not whitespace after the delimiter can be skipped. There are two methods used to detect these properties. The first method is used when quote characters are present in the file and detects adjacent occurrence of a quote character and another character (the potential delimiter). In this method the quote character and delimiter that occur most frequently are chosen. The second method is used when there are no quote characters in the file. In this case a frequency table is constructed that indicates how often a potential delimiter occurs and in how many rows (i.e. comma occurred $x$ times in $y$ rows). The character that most often matches the expected frequency is considered the delimiter, and a fallback list of preferred delimiters is used when a tie occurs. The detector also tries to detect whether or not double quoting is used within cells to escape a single quote character. This is done with a regular expression that can run into "catastrophic backtracking" for CSV files that end in many empty delimited rows. Therefore we place a timeout of two minutes on this detection method (normal operation never takes this long, so this restriction only captures this specific failure case). As mentioned, this method tries to detect when whitespace following the delimiter can be stripped. We purposefully do not include this in our method as the CSV specification states, "Spaces are considered part of a field and should not be ignored." (Shafranovich, 2005).

\footnotetext{
${ }^{7}$ The dialect sniffer was developed by Clifford Wells for his Python-DSV package (Wells, 2002) and was incorporated into Python version 2.3.
} 


\subsubsection{HypoParsr}

HypoParsr (Döhmen et al., 2017) is the first dedicated CSV parser that takes the problem of dialect detection and messy CSV files into account. ${ }^{8}$ The method uses a hierarchy of possible parser configurations and a set of heuristics to try to determine which configuration gives the best result. Unfortunately it is not possible use the HypoParsr $\mathrm{R}$ package to detect the dialect without running the full search that also includes header, table, and data type detection. Therefore, we run the complete program and extract the dialect from the outcome. This means however that both the running time and any potential failure of the method are affected by subsequent parsing steps and not just by the dialect detection. This needs to be kept in mind when reviewing the results. As the method can be quite slow, we add a timeout of 10 minutes per file. Finally, the quote character in the dialect is not always reported faithfully in the final parsing result, since the underlying parser can strip quote characters automatically. We developed our own method to check what quote character was actually used during parsing.

\subsubsection{Wrangler}

In Guo et al. (2011) a table suitability metric is presented that balances consistency of cell types against the number of empty cells and cells with potential delimiters in them. This can therefore be used to detect the dialect of CSV files by selecting the dialect that does best on this metric. The suitability metric uses the concept of column type homogeneity, i.e. the sum of squares of the proportions of each data type in a column. Since the exact type detection method used in the paper is not available, we use our type detection method instead.

\subsubsection{Variations}

In addition to our complete data consistency measure, we also consider several variations to investigate the effect of each component. Thus, we include a method that only uses the pattern score and one that only uses the type score. We also include a variation that does not use tie-breaking.

\subsection{Evaluation}

The methods are evaluated on the accuracy of the full dialect as well as on the accuracy of each component of the dialect. Note that a method can either fail by detecting the dialect incorrectly or it can fail by not returning a result at all. The latter case can happen due to a timeout or an exception in the code (for the Python Sniffer or HypoParsr), or due to a tie in the scoring measure (for the Wrangler suitability metric or our method). Both types of failure are considered to represent an incorrect dialect detection.

\subsection{Results}

We describe the results by focusing on dialect detection accuracy, robustness of the methods, accuracy on messy CSV files, and runtime. Unless explicitly stated otherwise, all results reflect performance on the test dataset.

\footnotetext{
${ }^{8}$ An $\mathrm{R}$ package for HypoParsr exists, but it was retracted from the $\mathrm{R}$ package repository on the request of the package maintainer. We nonetheless include the method in our experiments using the last available version.
} 


\begin{tabular}{lrrrrrrr} 
Property & HypoParsr & Sniffer & Suitability & Pattern & Type & No Tie & Full \\
\hline Delimiter & 87.32 & 86.95 & 65.57 & 92.67 & 88.02 & 91.50 & $\mathbf{9 4 . 9 1}$ \\
Quotechar & 83.03 & 92.47 & 45.02 & 95.38 & 90.21 & 93.95 & $\mathbf{9 7 . 3 5}$ \\
Escapechar & 87.83 & 94.34 & 74.78 & 97.95 & 96.24 & 95.57 & $\mathbf{9 9 . 2 2}$ \\
Overall & 80.61 & 85.66 & 38.60 & 91.16 & 83.38 & 90.72 & $\mathbf{9 3 . 7 6}$ \\
\hline
\end{tabular}

(a) GitHub corpus

\begin{tabular}{lrrrrrrr} 
Property & HypoParsr & Sniffer & Suitability & Pattern & Type & No Tie & Full \\
\hline Delimiter & 97.97 & 91.89 & 80.20 & 99.70 & 93.80 & 99.26 & $\mathbf{9 9 . 8 2}$ \\
Quotechar & 90.56 & 92.21 & 26.34 & 99.46 & 89.56 & 99.13 & $\mathbf{9 9 . 7 0}$ \\
Escapechar & 98.05 & 98.79 & 82.61 & $\mathbf{1 0 0 . 0 0}$ & 97.67 & 99.42 & 99.98 \\
Overall & 90.44 & 90.84 & 25.32 & 99.40 & 87.18 & 99.11 & $\mathbf{9 9 . 6 8}$ \\
\hline
\end{tabular}

(b) UKdata corpus

Table 1. Accuracy (in \%) of dialect detection for different methods on both corpora. Complete failure of a detection method is interpreted as an incorrect detection. "Pattern" and "Type" respectively indicate detection using only the pattern score or only the type score. "No Tie" indicates our method without tie-breaking.

\subsubsection{Detection Accuracy}

The accuracy of dialect detection is shown in Tables 1(a) and 1(b) respectively for the GitHub and UKdata corpora. We see that for both corpora and for all properties, our full data consistency method outperforms all alternatives. One exception occurs for the UKdata corpus, where the pattern-only score function yields a marginally higher accuracy on detecting the escape character. It is furthermore apparent from these tables that the GitHub corpus of CSV files is more difficult than the UKdata corpus. This is also reflected in the number of dialects observed in these corpora: 8 different dialects were found in the UKdata corpus vs. 35 in the GitHub corpus. We postulate that this difference is due to the nature of the creators of these files. CSV files from the UK government open data portal are often created using standard tools such as Microsoft Excel or LibreOffice, and therefore are more likely to adhere to the CSV format (Shafranovich, 2005). On the other hand, the creators of the files in the GitHub corpus are more likely to be programmers and data scientists who may use non-standard or custom-made tools for importing and exporting their CSV files and may therefore use different formatting conventions. It is interesting to note that even though the files in the UKdata corpus can be considered more "regular", we still achieve a considerable increase in detection accuracy over standard approaches.

Regarding the different variants of our method, we observe that the pattern score is in many cases almost as good as the full consistency measure. This confirms our earlier statement that the pattern score is the main driver of the method and that the type score serves mainly to further improve the accuracy. It is also clear that the type score alone does not suffice to accurately detect the dialect. The variant of our method that does not use tie-breaking yields a lower overall accuracy on both corpora, indicating the importance of tie-breaking in our method. Additional result tables are presented in Appendix $\mathrm{C}$ that show the accuracy separated by human vs. automatic detection of ground truth. Unsurprisingly, all methods perform better on files for which the dialect could be detected through automatic means than on those that required human annotation. 


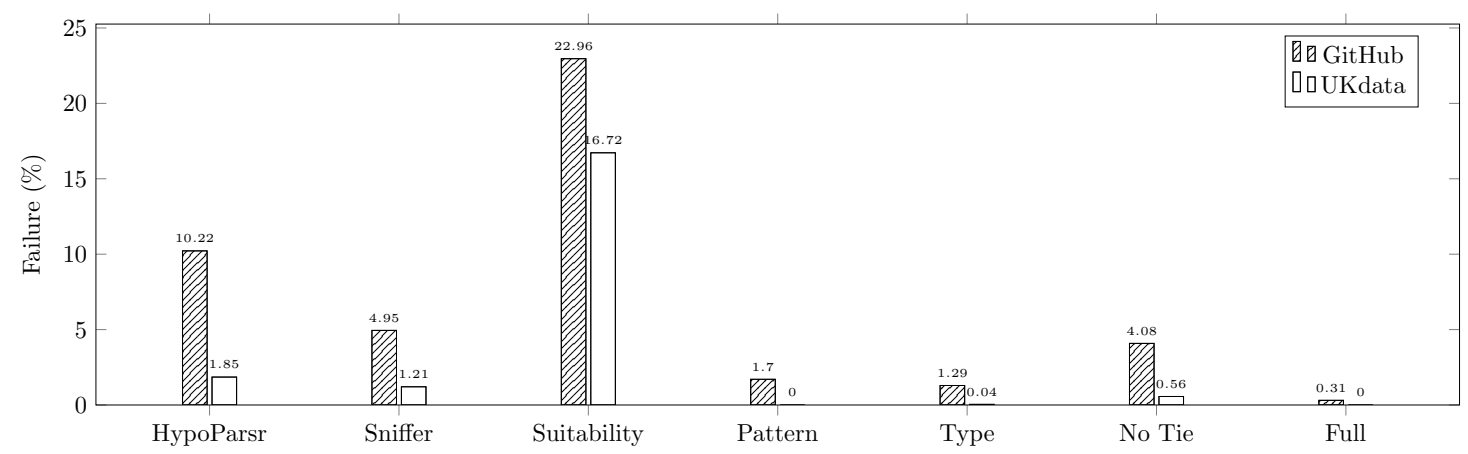

Figure 3. Percentage of failure for each of the detection methods on both corpora. Note that this shows how often the detection methods completely failed, not when it detected the parameters incorrectly.

\subsubsection{Failure}

Analysing the failure cases for the detection methods provides insight into their robustness. Figure 3 shows the proportion of files where no detection was made either due to a failure of the program or due to unbreakable ties in the scoring metric. This figure shows that HypoParsr (Döhmen et al., 2017) fails significantly more often on files from the GitHub corpus than files from the UKdata corpus. This can be due to the fact that HypoParsr was developed using files from the UKdata corpus, and because these files are generally easier to parse. However, since it is not possible to only use HypoParsr for dialect detection, it could also be the case that failures occur in a later stage of the parsing process. In fact, investigating the failure cases for this method further we observe that no result was obtained in $62.7 \%$ of the failures and that the timeout needed to be applied in 36.8\%. Similarly, for the Python Sniffer these values are 77.5\% and $22.5 \%$ respectively. Note further that our proposed method fails on no files in the UKdata corpus and on only $0.31 \%$ of files in the GitHub corpus. Failure in our method is only possible when ties occur, and the addition of both the type score and tie-breaking procedure ensures that this is rare. The high failure rate for the suitability metric from Guo et al. (2011) is exclusively due to ties in the scoring metric. Tie-breaking was not used here, as this is a feature of our method.

As Table 1(a) shows, an incorrect detection in our method occurs mostly due to an incorrect detection of the delimiter. Analysing these failure cases in more detail reveals that of the files where the delimiter was detected incorrectly, the majority of files had the comma as the delimiter. In these cases, an incorrect detection occurred mostly because our method predicted the space as the delimiter. Some of these files indeed had a regular pattern of whitespace in the cells and received a high type score as well, causing the confusion. Other files had the comma as true delimiter, but had only one column of data. In these cases the true comma delimiter could be deduced by the human annotator from a header or because certain cells that contained the comma were quoted, but this type of reasoning is not captured by the data consistency measure. In other failure cases, the pattern score predicted the correct delimiter, but the type score gave a low value, resulting in a low value of the consistency measure. Some of these failure cases can certainly be addressed by improving the type detection procedure. 


\begin{tabular}{lrrrrrrr} 
& HypoParsr & Sniffer & Suitability & Pattern & Type & No Tie & Full \\
\hline Standard (3890) & 85.84 & 91.13 & 44.45 & 93.29 & 86.22 & 93.55 & $\mathbf{9 5 . 8 4}$ \\
Messy (983) & 59.92 & 63.99 & 15.46 & 82.71 & 72.13 & 79.55 & $\mathbf{8 5 . 5 5}$ \\
Total (4873) & 80.61 & 85.66 & 38.60 & 91.16 & 83.38 & 90.72 & $\mathbf{9 3 . 7 6}$ \\
\hline
\end{tabular}

(a) GitHub corpus

\begin{tabular}{lrrrrrrr} 
& HypoParsr & Sniffer & Suitability & Pattern & Type & No Tie & Full \\
\hline Standard (4938) & 90.46 & 90.91 & 25.05 & 99.43 & 87.30 & 99.15 & $\mathbf{9 9 . 7 2}$ \\
Messy (31) & 87.10 & 80.65 & 67.74 & $\mathbf{9 3 . 5 5}$ & 67.74 & $\mathbf{9 3 . 5 5}$ & $\mathbf{9 3 . 5 5}$ \\
Total (4969) & 90.44 & 90.84 & 25.32 & 99.40 & 87.18 & 99.11 & $\mathbf{9 9 . 6 8}$ \\
\hline
\end{tabular}

(b) UKdata corpus

Table 2. Accuracy (in \%) of dialect detection for different methods on both corpora separated by standard and messy CSV files. The numbers in parentheses represent the number of files in each category. Complete failure of a detection method is again interpreted as an incorrect detection.

\subsubsection{Messy CSV Files}

Separating the files into those that are messy and those that follow the CSV standard further illustrates how our method improves over existing methods (see Table 2). CSV files are considered "standard" when they use the comma as the delimiter, use either no quotes or the " character as quote character, and do not use the escape character. The table also highlights the difference in the amount of non-standard files in the different corpora and reiterates that the GitHub corpus contains more non-standard CSV files. This table also emphasises the significance of our contribution, with an increase of $21.3 \%$ over the Python Sniffer for messy files averaged over both corpora. Note that on both corpora we also improve over current methods for standard files.

\subsubsection{Runtime}

Evaluating the runtime of each method is also relevant. Figure 4 shows violin plots for each method for both corpora. While the figures show that HypoParsr is the slowest detection method, this is not completely accurate. Since there is no way to use the method to only detect the dialect, the reported runtime is the time needed for the entire parsing process. The Python dialect sniffer is by far the fastest method and this can be most likely attributed to its simplicity in comparison to the other methods. Finally, all variations of our method have similar runtime characteristics and slightly outperform the Wrangler suitability metric. We note that our method has not been explicitly optimised for speed, and there are likely to be improvements that can be made in this aspect. Currently our method exhibits $O\left(|\mathbf{x}|\left|\Theta_{\mathbf{x}}\right|\right)$ complexity. Note however that the mean of the computation time for our method still lies well below one second, which is acceptable in almost all practical applications.

\section{Conclusion}

We have presented a data consistency measure for detecting the dialect of CSV files. This consistency measure emphasizes a regular pattern of cells in the rows and favours dialects that yield identifiable data types. While we apply the consistency measure only to CSV dialect detection in this paper, it is conceivable that there are other applications of this measure outside 


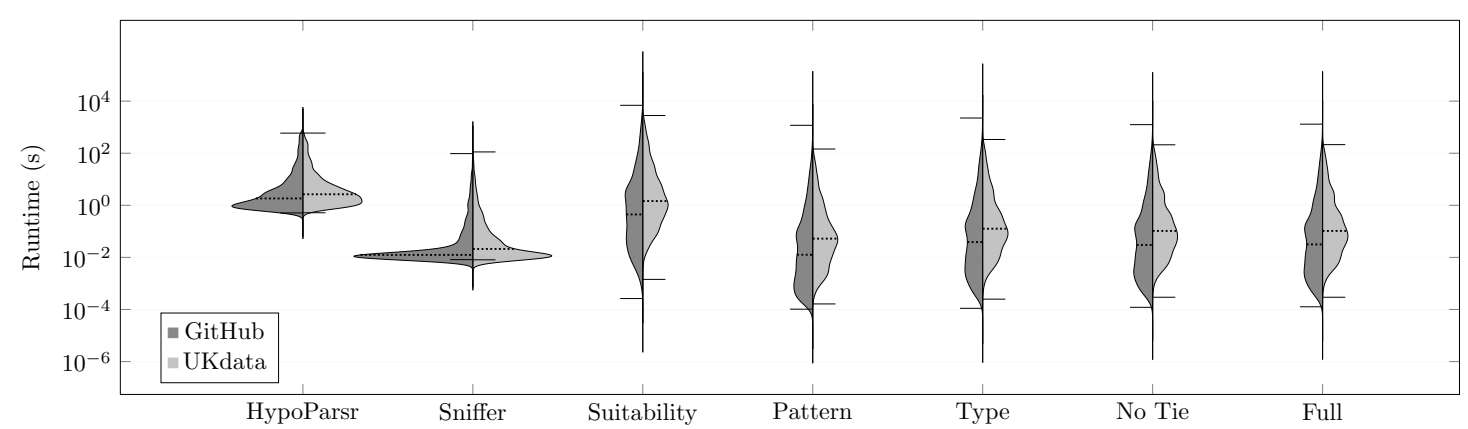

Figure 4. Runtime violin plots for both corpora. The whiskers show the minimum and maximum values and the dashed lines show the median. See the note on HypoParsr in the main text.

this area. For instance, one can imagine identifying unstructured tables in HTML documents or in free text, or use the measure to locate the tables within CSV or Excel files.

The main challenge for today's data scientists is the inordinate amount of time spent on preparing data for analysis. One of the difficulties they face is importing data from messy CSV files that often require manual inspection and reformatting before the data can be loaded from the file. In this paper we have presented a method for automatic dialect detection of CSV files that achieves near-perfect accuracy on a large corpus of real-world examples, and especially improves the accuracy on messy CSV files. This represents an important step towards automatically loading structured tabular data from messy sources. As such, it allows many data scientists to spend less time on mundane data wrangling issues and more time on extracting valuable knowledge from their data.

\section{Acknowledgements}

The authors would like to acknowledge the funding provided by the UK Government's Defence \& Security Programme in support of the Alan Turing Institute. The authors thank Chris Williams for useful discussions.

\section{References}

V. I. Arnold. Catastrophe Theory. Springer Science \& Business Media, 2003.

E. F. Codd. A relational model of data for large shared data banks. Communications of the $A C M, 13(6): 377-387,1970$.

D. Crockford. The application/json media type for javascript object notation (JSON). Technical Report RFC 4627, Internet Requests for Comments, 2006. URL www.rfc-editor.org/rfc/ rfc4627.txt.

Crowdflower. Data science report. http://visit.figure-eight.com/data-science-report. html, 2016.

T. Dasu and T. Johnson. Exploratory data mining and data cleaning, volume 479. John Wiley \& Sons, 2003. 
T. Döhmen, H. Mühleisen, and P. Boncz. Multi-hypothesis CSV parsing. In Proceedings of the 29th International Conference on Scientific and Statistical Database Management, pages 16:1-16:12. ACM, 2017. doi: 10.1145/3085504.3085520.

J. Eberius, C. Werner, M. Thiele, K. Braunschweig, L. Dannecker, and W. Lehner. DeExcelerator: a framework for extracting relational data from partially structured documents. In Proceedings of the 22nd ACM International Conference on Information 8 Knowledge Management, pages 2477-2480. ACM, 2013.

C. Evans. YAML draft 0.1, 2001. URL yaml.org.

K. Fisher, D. Walker, K. Q. Zhu, and P. White. From dirt to shovels: fully automatic tool generation from ad hoc data. In ACM SIGPLAN Notices, volume 43, pages 421-434. ACM, 2008.

Frictionless Data. CSV dialect specification, 2017. URL frictionlessdata.io/specs/ csv-dialect.

P. J. Guo, S. Kandel, J. M. Hellerstein, and J. Heer. Proactive wrangling: Mixed-initiative end-user programming of data transformation scripts. In Proceedings of the 24th annual ACM symposium on User interface software and technology, pages 65-74. ACM, 2011.

Kaggle. The state of data science \& machine learning, 2017. URL https://www.kaggle.com/ surveys/2017.

S. Kandel, A. Paepcke, J. Hellerstein, and J. Heer. Wrangler: Interactive visual specification of data transformation scripts. In Proceedings of the SIGCHI Conference on Human Factors in Computing Systems, pages 3363-3372. ACM, 2011.

S. C. Kleene. Representation of events in nerve nets and finite automata. In C. E. Shannon and J. McCarthy, editors, Automata Studies. Princeton University Press, 1956.

E. Koci, M. Thiele, Ó. Romero Moral, and W. Lehner. A machine learning approach for layout inference in spreadsheets. In IC3K 2016: Proceedings of the 8th International Joint Conference on Knowledge Discovery, Knowledge Engineering and Knowledge Management: volume 1: $K D I R$, pages 77-88, 2016.

S. Li and K. Momoi. A composite approach to language/encoding detection. In Proc. 19th International Unicode Conference, pages 1-14, 2001.

S. Lohr. For big-data scientists, "janitor work" is key hurdle to insights. The New York Times, Aug 2014. URL https://www.nytimes.com/2014/08/18/technology/ for-big-data-scientists-hurdle-to-insights-is-janitor-work.html.

J. Mitlöhner, S. Neumaier, J. Umbrich, and A. Polleres. Characteristics of open data CSV files. In 2nd International Conference on Open and Big Data (OBD), pages 72-79, Aug 2016. doi: 10.1109/OBD.2016.18.

H. T. Ng, C. Y. Lim, and J. L. T. Koo. Learning to recognize tables in free text. In Proceedings of the 37th annual meeting of the Association for Computational Linguistics on Computational Linguistics, pages 443-450. Association for Computational Linguistics, 1999.

D. Pinto, A. McCallum, X. Wei, and W. B. Croft. Table extraction using conditional random fields. In Proceedings of the 26th annual international ACM SIGIR conference on Research and development in information retrieval, pages 235-242. ACM, 2003. 
E. S. Raymond. The art of Unix programming. Addison-Wesley Professional, 2003.

J. Rovegno and M. Fenner. CSVY: YAML frontmatter for CSV file format, 2015. URL csvy . org/.

Y. Shafranovich. Common format and MIME type for comma-separated values (CSV) files. Technical Report RFC 4180, Internet Requests for Comments, 2005. URL https://tools. ietf.org/html/rfc4180.

C. Sutton, T. Hobson, J. Geddes, and R. Caruana. Data diff: Interpretable, executable summaries of changes in distributions for data wrangling. In Proceedings of the 24th ACM SIGKDD International Conference on Knowledge Discovery $\&$ Data Mining, pages 2279-2288. ACM, 2018.

J. Tennison. CSV on the web: A primer. W3C note, W3C, feb 2016. http://www.w3.org/TR/2016/NOTE-tabular-data-primer-20160225/.

J. Tennison and G. Kellogg. Metadata vocabulary for tabular data. W3C recommendation, W3C, dec 2015. http://www.w3.org/TR/2015/REC-tabular-metadata-20151217/.

The Unicode Consortium. The Unicode Standard, Version 11.0.0. 2018.

C. Wells. Python-DSV. http://python-dsv.sourceforge.net/, 2002. Accessed: 2018-11-08.

H. Wickham. Tidy data. Journal of Statistical Software, 59(10):1-23, 2014.

\section{A Data Type Detection}

As mentioned in the main text, we use a regular expression based type detection engine. Below is a brief overview of the different types we consider and the detection method we use for that type. The order of the types corresponds to the order in which we evaluate the type tests, and we stop when a matching type is found.

\section{Empty Strings}

Empty strings are considered a known type.

\section{URLs and Email Addresses}

For this we use two separate regular expressions.

\section{Numbers}

We consider two different regular expressions for numbers. First, we consider numbers that use "digit grouping", i.e. numbers that use a period or comma to separate groups of thousands. In this case we allow numbers with a comma or period as thousands separator and allow for using a comma or period as radix point, respectively. Numbers in this form can not have E-notation, but can have a leading sign symbol. The second regular expression captures the numbers that do not use digit grouping. These numbers can have a leading sign symbol (+ or -), use a comma or period as radix point, and can use E-notation (i.e. 123e10). The exponent in the E-notation can have a sign symbol as well. 
Time

Times are allowed in HH:MM:SS, HH:MM, and H:MM format. The AM/PM quantifiers are not included.

\section{Percentage}

This corresponds to a number combined with the $\%$ symbol.

\section{Currency}

A currency value is a number preceded by a symbol from the Unicode Sc category (The Unicode Consortium, 2018).

\section{Alphanumeric}

An alphanumeric string can follow two alternatives. The first alternative consists of first one or more number characters, then one or more letter characters, and then zero or more numbers, letters, or special characters. An example of this is the string 3 degrees. The second alternative first has one or more letter characters and then allows for zero or more numbers, letters, or special characters. An example of this is the string NW1 2DB. In both alternatives the allowed special characters are space, period, exclamation and question mark, and parentheses, including their international variants.

\section{$\mathbf{N} / \mathbf{A}$}

While nan or NaN are caught in the alphanumeric test, we add here a separate test that considers $\mathrm{n} / \mathrm{a}$ and $\mathrm{N} / \mathrm{A}$.

\section{Dates}

Dates are strings that are not numbers and that belong to one of forty different date formats. These date formats allow for the formats (YY) YYX (M) Mx (D)D, (D)Dx (M) Mx (YY) YY,

(M) Mx (D) Dx (YY) YY where $\mathrm{x}$ is a separator (dash, period, or space) and parts within parentheses can optionally be omitted. Additionally, the Chinese/Japanese date format

(YY)YY年 (M)M月 (D)D日 and the Korean date format (YY)YY년 (M)M월 (D)D일 are included.

\section{Combined date and time}

These are formats for joint date and time descriptions. For these formats we consider $<$ date $><$ time $>$ and $<$ date $>$ T<time $>$ as well as those with a time zone offset appended.

\section{B Additional Algorithm Descriptions}

Below we explicitly provide algorithms for functions mentioned in the main text that were omitted due to space constraints.

\section{B.1 Selecting Potential Delimiters}

Algorithm 3 gives the function for selecting potential delimiters of the file $\mathbf{x}$. The function FilterURLs replaces URLs in the file with a single letter, to avoid URLs generating spurious 
potential delimiters. We use $\mathcal{B}=\{., /$, ", ' $\}$ and $\mathcal{C}=\{\mathrm{Lu}, \mathrm{Ll}, \mathrm{Lt}, \mathrm{Lm}, \mathrm{Lo}, \mathrm{Nd}, \mathrm{Nl}$, No, Ps, Pe, Cc, Co $\}$.

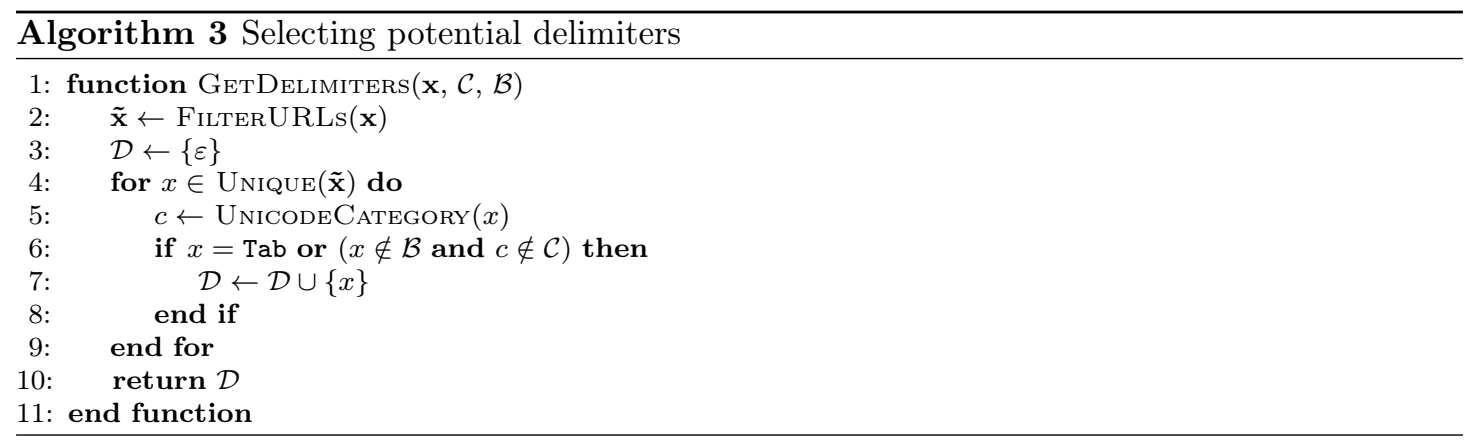

\section{B.2 Selecting Potential Quote Characters}

The function FilterURLs is as described in the previous section.

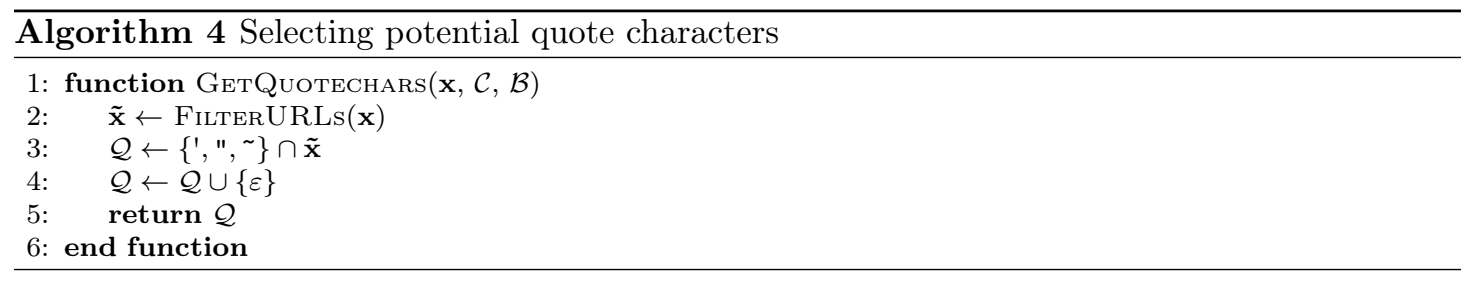

\section{B.3 Selecting Potential Escape Characters}

Potential escape characters are those in the Unicode Po (punctuation, other) category that are not explicitly blocked from being considered. For the latter we use a set $\mathcal{W}=\left\{!, ?, ",{ }^{\prime}, .,,,, ;,:, \backslash \%, *, \&, \#\right\}$.

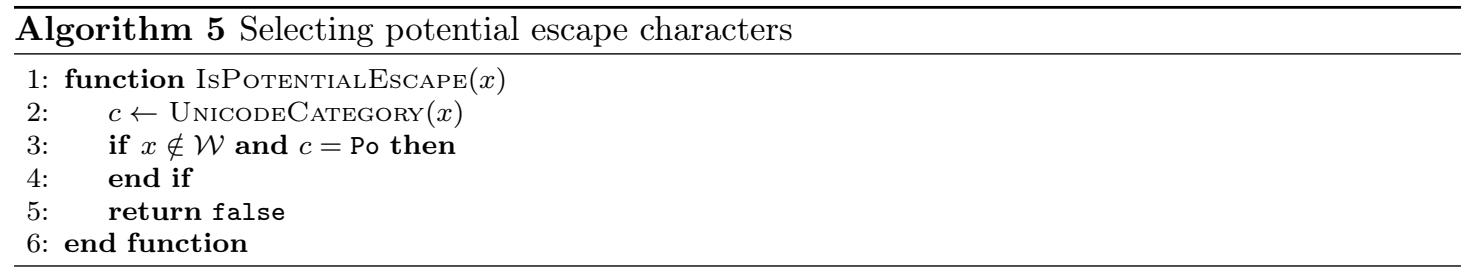

\section{B.4 Masked by Quote Character}

The function MaskedByQuote in Algorithm 2 is used to prune the list of potential dialects by removing those where the delimiter never occurs outside a quoted environment. This function is straightforward to implement: it iterates over all characters in the file and keeps track of where a quoted section starts and ends, while taking quote escaping into account. Given this it is straightforward to check whether the given delimiter always occurs inside a quoted section or not. 


\section{B.5 Parser}

The code we use for our CSV parser $f_{\boldsymbol{\theta}}^{-1}$ borrows heavily from the CSV parser in the Python standard library, but differs in a few small but significant ways. First, our parser only interprets the escape character if it proceeds the delimiter, quote character, or itself. In any other case the escape character serves no purpose and is treated as any other character and is not dropped. Second, our parser only strips quotes from cells if they surround the entire cell, not if they occur within cells. This makes the parser more robust against misspecified quote characters. Finally, when we are in a quoted cell we automatically detect double quoting by looking ahead whenever we detect a quote, and checking if the next character is also a quote character. This enables us to drop double quoting from our dialect and only marginally affects the complexity of the code.

\section{Additional Results}

\begin{tabular}{lrrrrrrr} 
Property & HypoParsr & Sniffer & Suitability & Pattern & Type & No Tie & Full \\
\hline Delimiter & 83.09 & 82.15 & 59.94 & 89.71 & 84.41 & 88.26 & $\mathbf{9 3 . 1 5}$ \\
Quotechar & 76.41 & 89.50 & 39.99 & 93.49 & 85.89 & 91.16 & $\mathbf{9 6 . 0 5}$ \\
Escapechar & 83.87 & 92.44 & 73.84 & 97.10 & 94.80 & 93.45 & $\mathbf{9 8 . 7 2}$ \\
Overall & 72.56 & 80.09 & 29.46 & 87.51 & 76.92 & 87.04 & $\mathbf{9 1 . 3 3}$ \\
\hline
\end{tabular}

(a) GitHub - human annotated

\begin{tabular}{lrrrrrrr} 
Property & HypoParsr & Sniffer & Suitability & Pattern & Type & No Tie & Full \\
\hline Delimiter & 93.87 & 94.40 & 74.29 & 97.28 & 93.61 & 96.54 & $\mathbf{9 7 . 6 4}$ \\
Quotechar & 93.30 & 97.07 & 52.83 & 98.32 & 96.91 & 98.27 & $\mathbf{9 9 . 3 7}$ \\
Escapechar & 93.98 & 97.28 & 76.23 & 99.27 & 98.48 & 98.85 & $\mathbf{1 0 0 . 0 0}$ \\
Overall & 93.09 & 94.29 & 52.77 & 96.81 & 93.40 & 96.44 & $\mathbf{9 7 . 5 4}$ \\
\hline
\end{tabular}

(b) GitHub - automatically annotated

\begin{tabular}{lrrrrrrr} 
Property & HypoParsr & Sniffer & Suitability & Pattern & Type & No Tie & Full \\
\hline Delimiter & 97.31 & 92.17 & 81.91 & 99.59 & 93.16 & 98.95 & $\mathbf{9 9 . 7 4}$ \\
Quotechar & 87.46 & 92.93 & 24.61 & 99.30 & 87.49 & 98.77 & $\mathbf{9 9 . 5 6}$ \\
Escapechar & 97.43 & 99.65 & 85.21 & $\mathbf{1 0 0 . 0 0}$ & 96.76 & 99.18 & 99.97 \\
Overall & 87.28 & 91.14 & 23.12 & 99.21 & 84.71 & 98.74 & $\mathbf{9 9 . 5 3}$ \\
\hline
\end{tabular}

(c) UKdata - human annotated

\begin{tabular}{lrrrrrrr} 
Property & HypoParsr & Sniffer & Suitability & Pattern & Type & No Tie & Full \\
\hline Delimiter & 99.42 & 91.28 & 76.42 & 99.94 & 95.22 & 99.94 & $\mathbf{1 0 0 . 0 0}$ \\
Quotechar & 97.42 & 90.63 & 30.17 & 99.81 & 94.12 & 99.94 & $\mathbf{1 0 0 . 0 0}$ \\
Escapechar & 99.42 & 96.90 & 76.87 & $\mathbf{1 0 0 . 0 0}$ & 99.68 & 99.94 & $\mathbf{1 0 0 . 0 0}$ \\
Overall & 97.42 & 90.18 & 30.17 & 99.81 & 92.64 & 99.94 & $\mathbf{1 0 0 . 0 0}$ \\
\hline
\end{tabular}

(d) UKdata - automatically annotated

Table 3. Accuracy (in \%) of dialect detection for different methods on both corpora, split between files that were manually annotated and files that where the dialect was determined through automatic means. As in the main text, failure of a method to return a result is interpreted as an incorrect detection. 